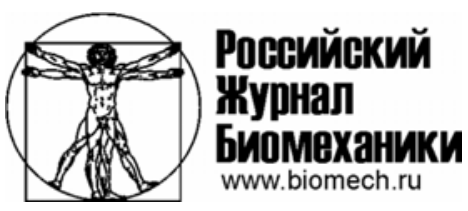

\title{
ЧИСЛЕННОЕ ИССЛЕДОВАНИЕ ВЛИЯНИЯ СТЕПЕНИ НЕДОПОКРЫТИЯ ВЕРТЛУЖНОГО КОМПОНЕНТА НА НЕСУЩУЮ СПОСОБНОСТЬ ЭНДОПРОТЕЗА ТАЗОБЕДРЕННОГО СУСТАВА
}

\author{
Ю.Г. Коноплев ${ }^{1}$, А.В. Мазуренко ${ }^{2}$, О.А. Саченков ${ }^{1}$, Р.М. Тихилов ${ }^{3}$ \\ ${ }^{1}$ Институт математики и механики имени Н.И. Лобачевского Казанского (Приволжского) федерального \\ университета, Россия, 420008, Казань, ул. Кремлевская, 18, e-mail: 4works@bk.ru \\ 2 Травматологическо-ортопедическое отделение Федерального центра травматологии, ортопедии и \\ эндопротезирвоания Минздрава России, Россия, 428020, Чебоксары, ул. Федора Гладкова, 33, e-mail: \\ amazourenko.home@inbox.ru \\ ${ }^{3}$ Российский научно-исследовательский институт травматологии и ортопедии им. Р.Р. Вредена \\ Минздрава России, Россия, 195427, Санкт-Петербург, ул. Академика Байкова, 8
}

\begin{abstract}
Аннотация. Целью работы является определение критической величины недопокрытия ацетабулярного компонента, при которой возможна его имплантация без использования дополнительной опоры. Актуальность данного исследования продиктована клинической практикой и отсутствием обоснованных рекомендаций для тактики эндопротезирования с недопокрытием вертлужного компонента. Численные исследования выполнены с помощью метода конечных элементов в пакете Siemens NX. Степень дисплазии оценивалась на основе оригинального способа интраоперационного измерения площади недопокрытия вертлужного компонента. Были проведены расчеты для чашек диаметром 52, 54 и 56 мм с учетом влияния контакного взаимодействия. Для решения задачи напряженно-деформиуремого тела использовался метод конечных элементов. Численный алгоритм реализации контактного взаимодействия основан на итерационном процессе определения зоны контакта. На основе результатов численных расчетов был изучен механизм потери несущей способности ацетабулярного компонента, получены зависимости величины критической реакции в суставе от величины недопокрытия. Под критической величиной суставной реакции понималась наименьшая сила, при которой происходит потеря несущей способности, отождествляемая с появлением относительного скольжения в области контакта. Была выявлена стабильность имплантата при степени недопокрытия до $20 \%$; от 20 до $30 \%$ - величина критической силы превышает 2 кН и уменьшается с увеличением процента недопокрытия. Полученные данные сравнивались с клиническими и экспериментальными, разница в определяемых величинах составляла порядка 1 кН (порядка 35 \% в относительных величинах). На основании полученных данных было получено экспоненциальное приближение зависимости величины критической реакции в суставе от различной степени недопокрытия. Полученная зависимость величины допустимого недопокрытия от массы пациента позволяет хирургу индивидуально и объективно оценить возможность установки ацетабулярного компонента эндопротеза.
\end{abstract}

Ключевые слова: эндопротезирование тазобедренного сустава, дисплазия, контактное взаимодействие, математическое моделирование.

(C) Коноплев Ю.Г., Мазуренко А.В., Саченков О.А., Тихилов Р.М., 2015

Коноплев Юрий Геннадьевич, д.ф.-м.н., профессор, академик Российской академии естественных наук и академии наук Республики Татарстан, заведующий кафедрой теоретической механики, Казань

Мазуренко Андрей Васильевич, заведующий травматолого-ортопедическим отделением, врач травматолог-ортопед, Чебоксары

Саченков Оскар Александрович, к.ф.-м.н., научный сотрудник института математики и механики имени Н.И. Лобачевского, Казань

Тихилов Рашид Муртузалиевич, д.м.н., профессор, директор Российского научно-исследовательского института травматологии и ортопедии им. Р.Р. Вредена Минздрава России, Санкт-Петербург 


\section{ВВеДЕНИЕ}

В настоящее время ведётся дискуссия по поводу тактики эндопротезирования тазобедренного сустава при тяжёлых дисплазиях вертлужной впадины. Остаются актуальными вопросы об уровне установки вертлужного компонента $[11,16,18,19$, 25], необходимости резекции или остеотомии проксимального конца бедренной кости, возможности двухэтапного эндопротезирования тазобедренного сустава. Существуют различные варианты установки вертлужного имплантата, в том числе и с частичным недопокрытием вертлужного компонента (рис. 1), например: частичное недопокрытие вертлужного компонента; вертикальное положение чашки эндопротеза; смещение его в краниальном направлении на расстояние до трех см выше уровня «фигуры слезы»; применение опорных и антипротрузионных конструкций (типа Muller, BurchSchneider); костная пластика массивным спонгиозным трансплантатом. Каждый из перечисленных вариантов имеет свои преимущества и недостатки, возможна комбинация различных способов. Всё вышеперечисленное свидетельствует о значительном арсенале методов и их комбинаций и отсутствии на сегодняшний день оптимальной тактики при эндопротезировании тазобедренного сустава у пациентов с диспластическим коксартрозом и указывает на настоятельную необходимость поиска современных решений. Современные методы математического моделирования позволяют производить расчеты биомеханического поведения сустава как трехмерного тела $[2,8-11,14]$, в том числе определять области применения различных тактик эндопротезирования на основе проведенных расчетов.

Площадь недопокрытия оценивалась согласно методике, описанной в патенте на изобретение РФ № 2412646 от 27.02.2011 «Способ определения степени покрытия вертлужного компонента бесцементной фиксации в процентном соотношении после его имплантации в обработанную вертлужную впадину при первичных и ревизионных операциях эндопротезирования тазобедренного сустава».

Целью настоящего исследования является определение критической величины недопокрытия ацетабулярного компонента, при которой возможна его имплантация без использования дополнительной опоры. Для этого была разработана численная
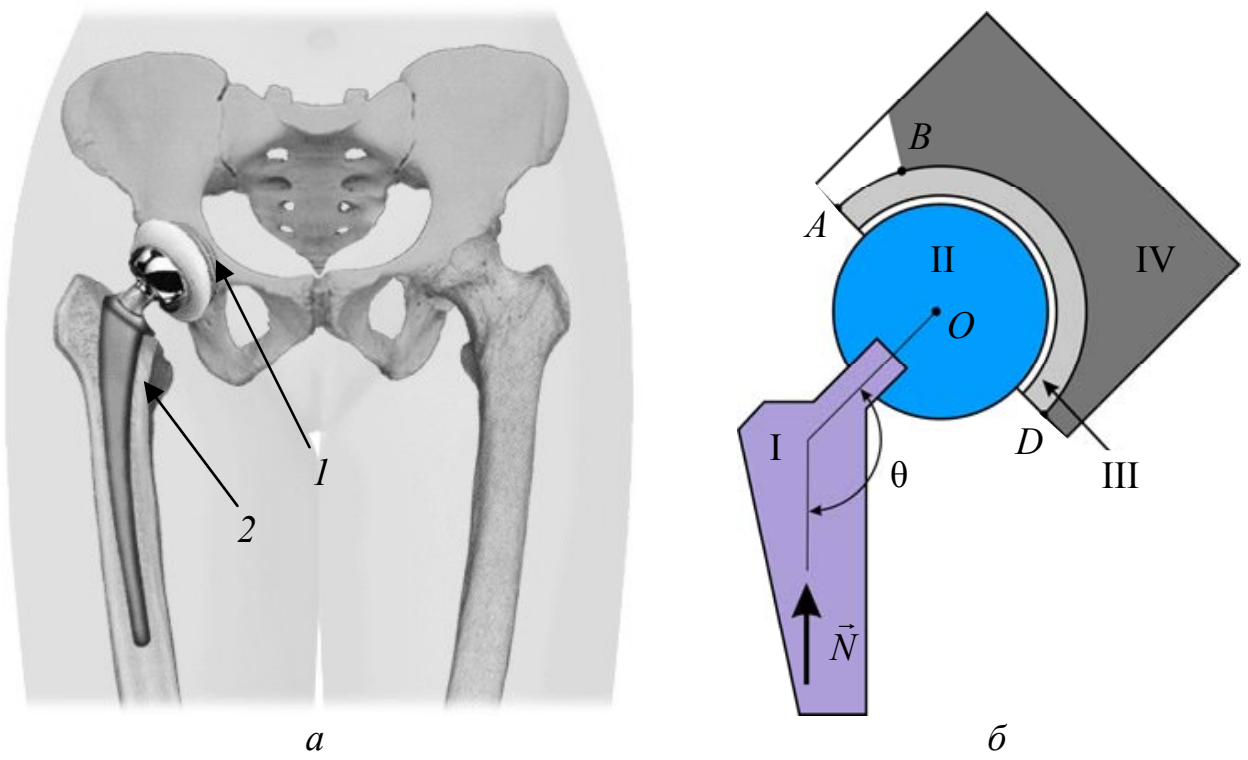

Рис. 1. Эндопротез: $a$ - изображение установки ( 1 - вертлужный компонент; 2 - бедренный компонент); $\sigma$ - расчетная схема (I - бедренный компонент; II, III - вертлужный компонент (II - головка, III - чашка); IV - таз; дуга $A D$ вертлужный компонент; дуга $A B$ - область недопокрытия; $O$ - центр головки бедренного компонента; $N$ - реакция бедренного компонента эндопротеза) 
реализация контактного взаимодействия для конечно-элементной модели, были проведены численные исследования влияния степени недопокрытия вертлужного компонента на несущую способность имплантата и определение критической силы для различной степени недопокрытия.

\section{МАТЕРИАЛЫ И МЕТОДЫ}

\section{Постановка задачи}

\section{Принятые допущения}

Силы, действующие на вертлужный компонент (см. рис. 1), передаются через бедренный компонент имплантата, а значит, при движении изменяются как количественно, так и по направлению действия (при ходьбе расчетное значение реакции, возникающей в суставе, превышает вес человека в 2-3 раза $[2,10,14])$. В работе учитывалась статическая составляющая усилия, что может быть обосновано малостью амплитуды динамической компоненты.

В математической модели рассматривалась вертлужная часть эндопротеза, бедренный компонент (I на рис. 1) заменялся силовым влиянием. Так, на рис. 2 угол $\theta$ (угол между нормалью к поверхности $A_{1} D_{1}$, проходящий через центр $O$, и направлением ножки эндопротеза $E F$ ) характеризует угол наклона ножки эндопротеза, он необходим для пересчета сил, заменяющих бедренный компонент. Натяг при установке тазовой компоненты эндопротеза не учитывался ввиду малости из-за недопокрытия. Для моделирования силового влияния бедренного компонента была поставлена следующая задача.

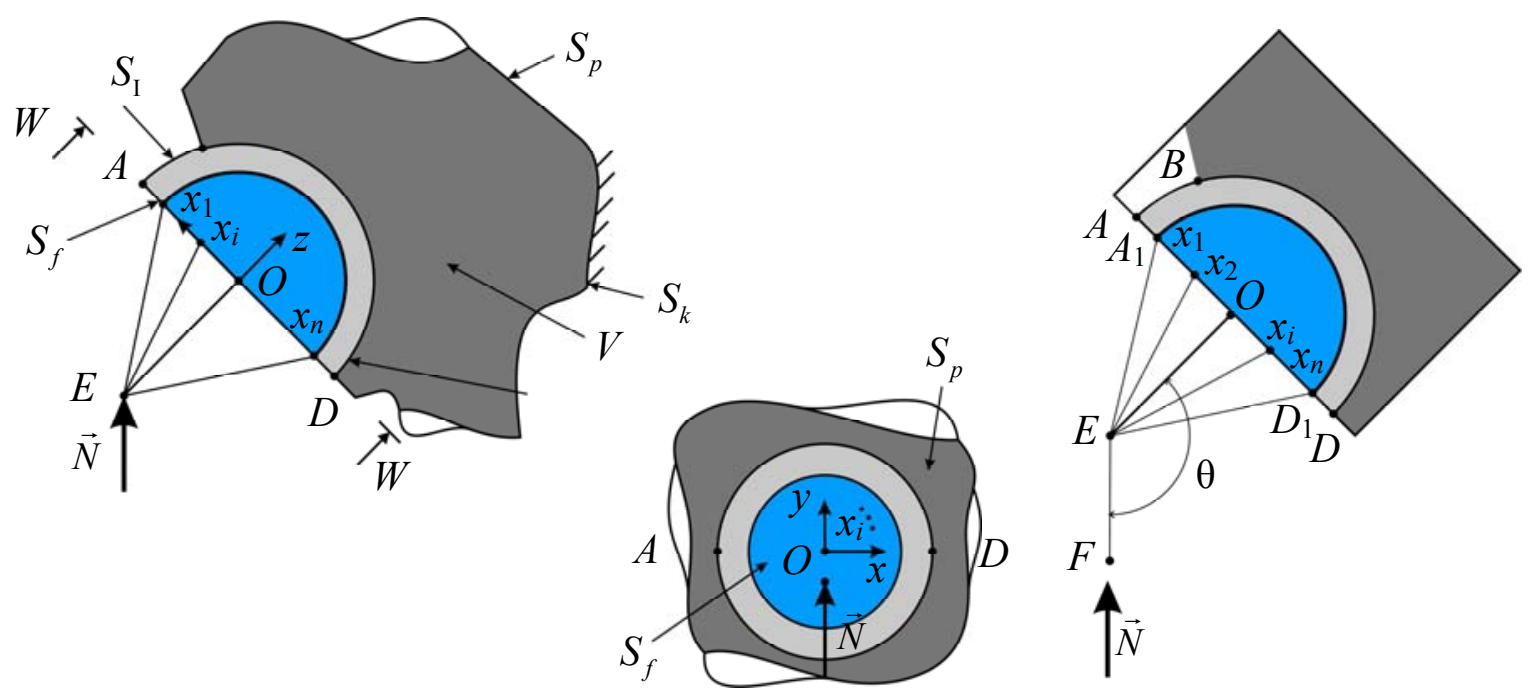

Рис. 2. Расчетная схема: $S_{\text {I }}$ - поверхность имплантата (дуга $A D$ - чашка вертлужного компонента, дуга $A_{1} D_{1}$ - головка вертлужного компонента); $S_{p}-$ полная поверхность бедренной кости; $S_{p} \cap S_{\text {I }}$ - поверхность контакта кости с имплантатом (дуга $B D$ ); $S_{\mathrm{I}} \backslash\left(S_{p} \cap S_{\mathrm{I}}\right)$ - область недопокрытия (дуга $\left.A B\right) ; O$ центр головки вертлужного компонента; $N$ - реакция бедренного компонента эндопротеза; $E F$ - направление ножки бедренного компонента; $\theta$ - угол наклона шейки бедренного компонента; $S_{f}$ - поверхность со статическими граничными условиями, $S_{k}-$ поверхность с кинематическими граничными условиями 
На поверхности $A_{1} D_{1}$ размещалось $n$ точек (точки с радиусом-вектором $\vec{X}_{i}\left(X_{i}^{x}, X_{i}^{y}, 0\right) \in S_{f}$ на рис. 2), которые соединялись абсолютно жесткими стержнями с точкой $E$ (см. рис. 2). Расстояние $O E$ задавалось равным расстоянию между центром $O$ и линией действия силы $\vec{N}$ (см. рис. 1 ), и к точке $E$ прикладывалась указанная сила $\vec{N}$. Положение точки $E$ однозначно описывается двумя параметрами: углом $\theta$ и расстоянием $O E$. После этого для полученной ферменной конструкции определялись реакции $\vec{R}_{i}\left(R_{i}^{x}, R_{i}^{y}, R_{i}^{z}\right)$ в точках $\vec{X}_{i}$, при этом соблюдались условия статического равновесия:

$$
N \cos \theta=\sum_{i=1}^{n} R_{i}^{z}, N \sin \theta=\sum_{i=1}^{n} R_{i}^{y}, 0=\sum_{i=1}^{n} R_{i}^{x} .
$$

Определение этих реакций было реализовано в методе конечных элементов в пакете Siemens $N X$. При численной реализации для преодоления статической неопределимости полученной ферменной конструкции стержням задавался значительно превышающий модуль Юнга (порядка $10^{20}$ Па). Найденные реакции и являются статическими граничными условиями, при конечно-элементной реализации разбиение областей производилось таким образом, чтобы в каждой точке $\vec{X}_{i}$ находился узел.

\section{Уравнения задачи}

Механическое поведение системы, занимающей область $V$ в $R^{3}$ с границей $S$, в рамках линейной теории упругости описывается следующей системой уравнений:

$$
\begin{gathered}
\nabla \cdot \tilde{\sigma}=0, \forall \mathbf{x} \in V, \\
\tilde{\varepsilon}=\frac{1}{2}\left(\nabla \vec{u}+(\nabla \vec{u})^{T}\right), \forall \mathbf{x} \in \bar{V}, \\
\tilde{\sigma}=\tilde{\tilde{E}}: \tilde{\varepsilon}, \forall \mathbf{x} \in \bar{V}, \\
\vec{u}=0, \forall \mathbf{x} \in S_{k}, \\
\vec{n} \cdot \tilde{\sigma}=\vec{R}\left(\vec{X}_{i}\right), i=\overline{1, n}, \forall \vec{X}_{i} \in S_{f},
\end{gathered}
$$

где $\bar{V}=V \cup S, S=S_{k} \cup S_{f}, \vec{u}$ - вектор перемещений, $\tilde{\sigma}-$ тензор напряжений; $\tilde{\varepsilon}-$ тензор упругих деформаций; $S_{f}-$ поверхность со статическими граничными условиями; $S_{k}$ - поверхность с кинематическими граничными условиями.

Для оценки напряженно-деформированного состояния костной ткани важно учитывать контактное взаимодействие между имплантатом и костью, существуют различные подходы для реализации контактного взаимодействия $[3,6,7,12,27]$, за основу был взят метод из работ $[3,27]$, в котором для реализации контактного взаимодействия используются следующие соотношения: кинематические уравнения, условия контактного взаимодействия, уравнение статического равновесия.

В ходе деформации исследуемой модели происходит движение точки исходной грани (точка D) относительно целевой грани. Под исходной гранью понимается грань, принадлежащая более жесткому материалу (штампу), для нашей модели это чашка имплантата (рис. $3, a$ (тело $A$ )); под целевой - грань более податливого тела, для нашей модели это кость (рис. 3 , $a$ (тело $B)$ ). При нагружении точки тел $A$ и $B$ получают 
перемещения $\vec{u}$, тогда абсолютное положение этих точек представимо в виде $\vec{r}=\vec{r}^{0}+\vec{u}$, где $\vec{r}$ - текущее положение, $\vec{r}^{0}-$ начальное положение. Контактное взаимодействие тел происходит на некотором участке $S$ (см. рис. $3, a$ ), для точек вступивших в контакт (на рис. $3, a$ это точки $C$ и $D$ ), должно выполняться условие $\left.\left(\vec{r}_{H}-\vec{r}_{T}\right) \cdot \vec{n}\right|_{S}=0$, из которого следует

$$
\left(\vec{r}_{H}^{0}-\vec{r}_{T}^{0}\right) \cdot \vec{n}=\left.\left(\vec{u}_{H}-\vec{u}_{T}\right) \cdot \vec{n}\right|_{S},
$$

где $\vec{r}_{H}^{0}, \vec{u}_{H}$ - начальное положение и перемещение точки $D ; \vec{r}_{T}^{0}, \vec{u}_{T}-$ начальное положение и перемещение точки $C ; \vec{n}$ - нормаль к поверхности в точке.

На рис. 3 , б представлены свободные грани элементов целевой $(A)$ и исходной $(B)$ поверхностей. В процессе решения через точку $D$ (воздействующей поверхности) строится репер: тангенциальные векторы $\vec{e}_{1}, \vec{e}_{2}$ и нормаль $\vec{n}$. Пересечение нормали с целевой гранью происходит в точке $C$. При расчетах предполагается, что проникновение воздействующей точки в целевую грань проходит через точку $C$.

Величина проникновения рассчитывается по формуле

$$
p=p_{0}+\left(\vec{u}_{H}-\vec{u}_{T}\right) \vec{n},
$$

где $p_{0}$ - начальное проникновение.

В рамках конечно-элементной модели под контактной парой нужно понимать совокупность узлов, находящихся на исходной и целевой поверхностях. Тогда (7) можно выразить через интерполяционные функции для узлов исходной и целевой граней элементов в виде

$$
p=p_{0}+\left[q_{n}\right]\left(\begin{array}{l}
u_{H} \\
u_{T}
\end{array}\right),
$$

где $\left[q_{n}\right]$ - матрица-строка, состоящая из произведения интерполяционных функций на нормали, вид этих функций зависит от типа конечного элемента, $u_{H}, u_{T}$ - узловые перемещения исходной и целевой грани.

Аналогично для тангенциального скольжения можно получить выражение

$$
\Delta \xi=\xi_{0}+\left[q_{t}\right]\left(\begin{array}{l}
u_{H} \\
u_{T}
\end{array}\right) .
$$

Выписывая выражения (8), (9) для всех контактных элементов в матричном виде, получим

$$
\begin{gathered}
\{p\}=\left[Q_{n}\right]\{U\}+\left\{P_{0}\right\}, \\
\{\Delta \xi\}=\left[Q_{t}\right]\{U\}+\left\{\xi_{0}\right\} .
\end{gathered}
$$

При расчете матрицы жесткости определяются дополнительные слагаемые штрафные функции, связанные с влиянием контактного взаимодействия штампа (чашка вертлужной впадины) на костную ткань, так называемая контактная жесткость, для вычисления ее использовалось выражение

$$
K=e \cdot E \cdot d A,
$$

где $e-$ штрафная жесткость; $E$ - приведенный модуль упругости контактирующих материалов; $d A$ - площадь контактирующей поверхности.

Для учета сил трения используется закон Кулона:

$$
\left|t_{t}\right|-t_{n} f=0
$$

где $t_{t}$ - сила трения, $f$ - коэффициент трения. 


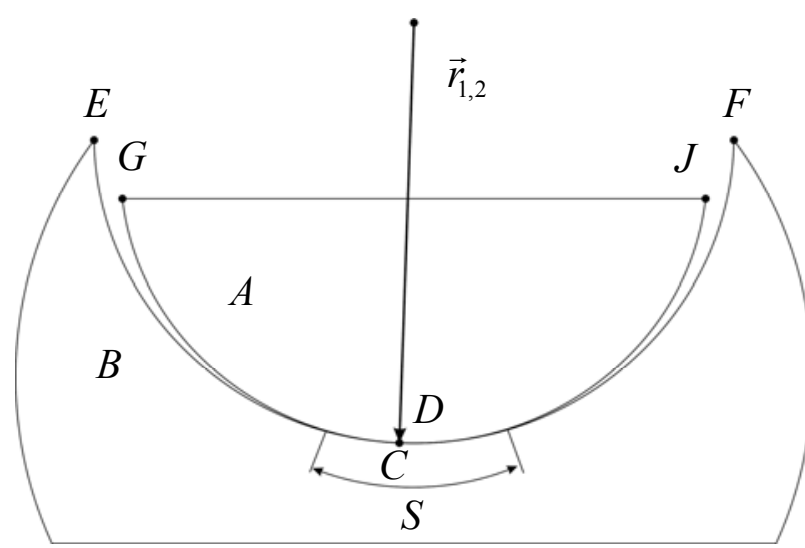

$a$

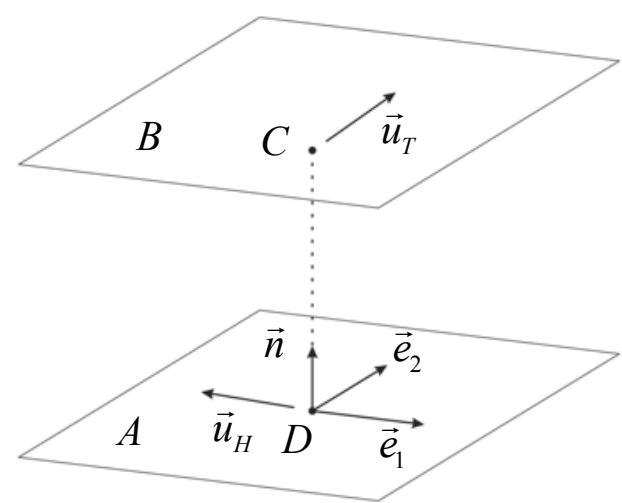

$\sigma$

Рис. 3. Исходная $(A)$ и целевая $(B)$ поверхности: $a-E F$ - поверхность вертлужной впадины; $G J$ - поверхность имплантата; $S$ - область контакта; $\vec{r}_{1}, \vec{r}_{2}$ - радиусвектор точки $D$ и $C$ соответственно; $\sigma-\vec{e}_{1}, \vec{e}_{2}$ - тангенциальные векторы; $\vec{n}-$ нормаль; $p_{0}-$ начальное проникновение, $\vec{u}_{H}-$ перемещение точки $D ; \vec{u}_{T}-$ перемещение точки $C$

При $\left|t_{t}\right| \leq t_{n} f$ - поверхности сцеплены, при $\left|t_{t}\right|>t_{n} f$ - происходит скольжение контактирующих поверхностей относительно друг друга.

Для определения линейного контактного взаимодействия используется расширенный метод Лагранжа [27]. Удовлетворение условия линейного контакта (нулевое взаимопроникновение) достигается корректировкой модулей контактных сил $t$ на каждой итерации алгоритма линейного контакта согласно выражению (6).

$$
t^{j}=t^{j-1}+e p,
$$

где $t$ - контактная сила; $p$ - текущее значение проникновения в точке; $e$ - штрафная жесткость.

Алгоритм определения линейного контактного взаимодействия:

1. Задание списка контактных элементов на основе конечно-элементной модели, под этим списком подразумевается список элементов, которые могут образовывать контактные пары.

2. Расчет жесткостных характеристик элементов и штрафных жесткостей контактных элементов.

3. Определение знаков сил взаимодействия элементов с учетом граничных статических и кинематических условий.

4. Запуск «Первого» цикла для определения статуса контактного элемента в зависимости от взаимопроникновения и контактного усилия, таким образом, получаем список контактных элементов (нахождение области контакта).

5. Добавление контактной жесткости (11) к глобальной матрице жесткости активных контактных элементов.

6. Запуск «Второго» цикла, где на каждой итерации определяется проникновение контактных пар и согласно выражению (6) в соответствии с заданной штрафной жесткостью вычисляются контактные силы. При достижении максимального количества итераций или сходимости контактных сил (разница контактных сил за шаг не превышает заданной точности) происходит возврат в «Первый» цикл. 
7. «Первый» цикл: обновление статуса контактных элементов с учетом подсчитанных контактных сил, проверка списка контактных элементов на нахождение в области контакта. Если обнаружено взаимопроникновение среди контактных элементов, которые не входят в список (пункт 4), то область контакта обновляется и алгоритм повторяется с пункта 5.

В этом случае механическое поведение системы, описываемое системой уравнений (1)-(5), дополняется следующими уравнениями. Для точек, вошедших в контакт, выполняется условие (14). На поверхности контакта тела взаимодействуют друг с другом, что приводит к возникновению нормального давления (15) и сил трения (16).

$$
\begin{gathered}
\left(\vec{r}_{P}^{0}-\overrightarrow{\mathrm{r}}_{I}^{0}\right) \cdot \vec{n}=\left(\vec{u}_{P}-\vec{u}_{I}\right) \cdot \vec{n}, \forall \mathbf{x} \in S_{p} \cap S_{I}, \\
\vec{t}_{n}=-t_{n} \vec{n}, t_{n}>0, \forall \mathbf{x} \in S_{p} \cap S_{I}, \\
\left|t_{t}\right|-t_{n} f \leq 0, \forall \mathbf{x} \in S_{p} \cap S_{I} .
\end{gathered}
$$

Основной задачей моделирования является определение зависимости между значением силы и площадью сегмента сферы (след этого сегмента соответствует дуге $A B$ на рис. 1), при котором в процессе эксплуатации будет проявляться нестабильность вертлужного компонента, т.е. потеря им несущей способности. Интерес представляет наименьшая сила, при которой наблюдается нестабильность, такую силу в дальнейшем будем называть критической силой.

\section{Численное решение задачи}

Для проведения численных исследований была построена трехмерная модель тазобедренного сустава, а также ацетабулярного компонента. На рис. 4 приведена построенная модель с дисплазией $(a)$ и конечно-элементное разбиение $(б)$. В расчетах учитывалось контактное взаимодействие между ацетабулярным компонентом и костной тканью с учетом сил трения $[2-5,9,12,23,27]$. В расчетах использовался четырехузловый тетраэдальный конечный элемент с линейной аппроксимацией.
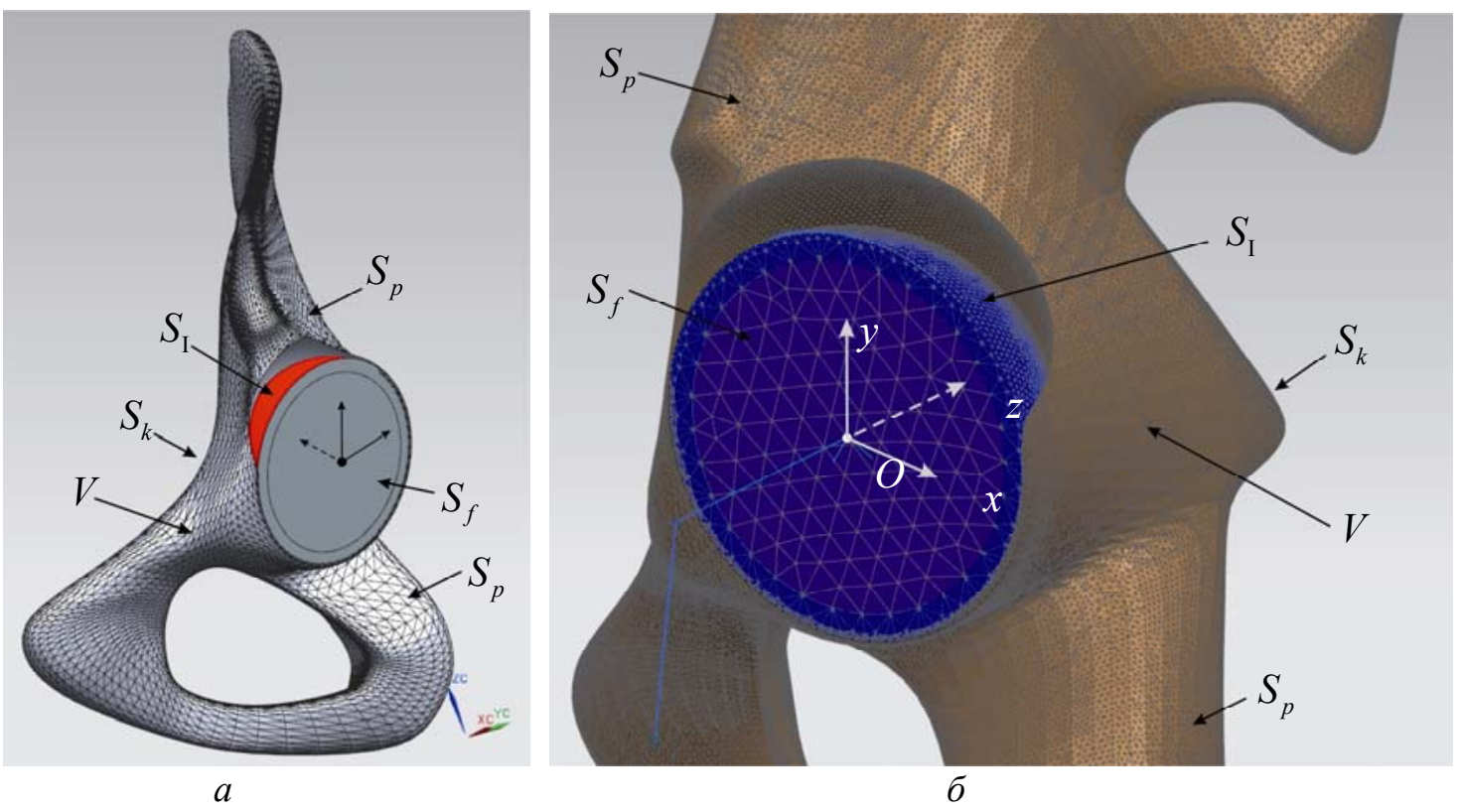

Рис. 4. Конечно-элементная аппроксимация расчетной области: $a$ - модель сустава c ацетабулярным компонентом (темным выделена область недопокрытия вертлужного компонента); $\sigma$ - конечно-элементное разбиение экваториального сечения ацетабулярного компонента эндопротеза 
Ацетабулярный компонент моделировался как двухкомпонентный объект, внешняя чашка титановая $(E=112$ ГПа, $v=0,3)$, внутренняя чашка керамическая ( $E=102$ ГПа, $v=0,26$, где $E$ - модуль Юнга, $v-$ коэффициент Пуассона). Характеристики кости: $E=6$ ГПа, $v=0,3$ [2], коэффициент трения между костной тканью и имплантатом принимался равным 0,95; между титановым и керамическим компонентами задавалось условие прилипания. Диаметр титановой чашки брался на 4 мм больше диаметра вертлужной впадины, проводились расчеты для чашек диаметром 52, 54, 56 мм. Дисплазия вертлужной впадины моделировалась сферическом вырезом (см. рис. $4, a$ ).

Для определения критической величины усилия применялся итерационный алгоритм, в рамках которого варьировалась прикладываемая сила и оценивалось перемещение вертлужного компонента в костной ткани. Для оценки потери несущей способности было использовано два критерия. В первом критерии оценивалось относительное перемещения узлов ацетабулярной чашки в контактной области (скольжение), т.е. выполнение условия $\left|t_{t}\right|>t_{n} f$; во втором критерии оценивались максимальные касательные напряжения в костной ткани вертлужной впадины,

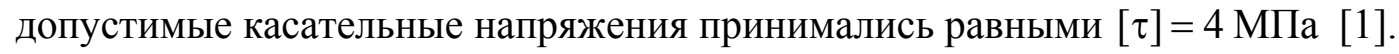

\section{РЕЗУЛЬТАТЫ И ОБСУЖДЕНИЕ}

Были проведены расчеты для величин недопокрытия в диапазоне от 15 до 50 \%. При величине недопокрытия до 18 \% вертлужный компонент вел себя стабильно при нагрузках вплоть до 4 кН. На рис. 5 показана величина вертикальной составляющей вектора перемещения вертлужного компонента при потере несущей способности $(a)$ и контактное давление (б) под действием силы $1000 \mathrm{H} \mathrm{с} \mathrm{недопокрытием} 30$ \%. Большие относительные перемещения узлов ацетабулярной чашки в контактной области говорят о потере несущей способности вертлужного компонента. На рис. $5, \sigma$ - резкий скачок контактного давления в области чашки (от 90 до $170 \mathrm{MПа),} \mathrm{что} \mathrm{связано} \mathrm{с}$ численной реализацией (скачок наблюдается на пересечении линии действия силы и области контакта).

Было отмечено изменение распределения контактного давления на вертлужном компоненте при изменении процента недопокрытия. Так, при недопокрытии до 30 \% основными зонами локализации напряжений являются область в центре чашки и полоса (см. рис. 6, a), проходящая около границы покрытия ацетабулярного
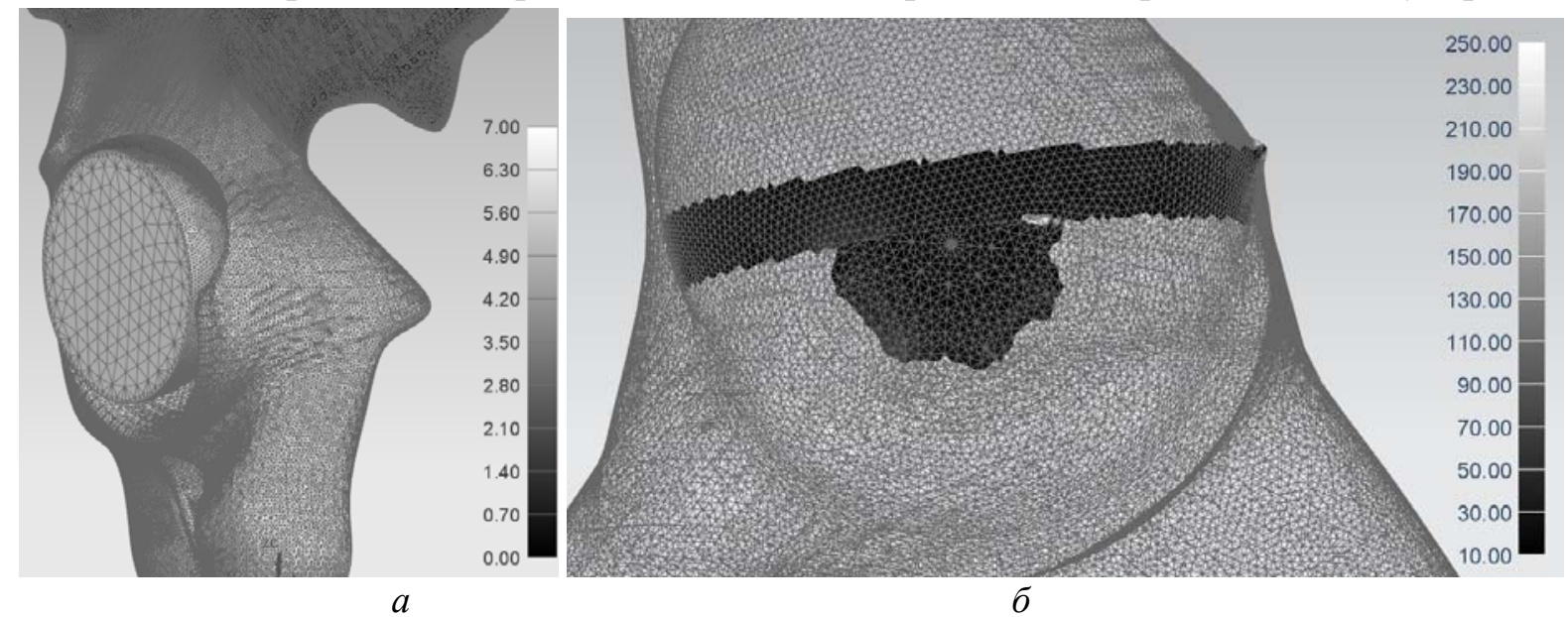

Рис. 5. Потеря несущей способности вертлужного компонента при недопокрытии $30 \%$ : $a$ - величина вертикальных перемещений чашки, мм; $\sigma$ - контактное давление вертлужной впадины, МПа 


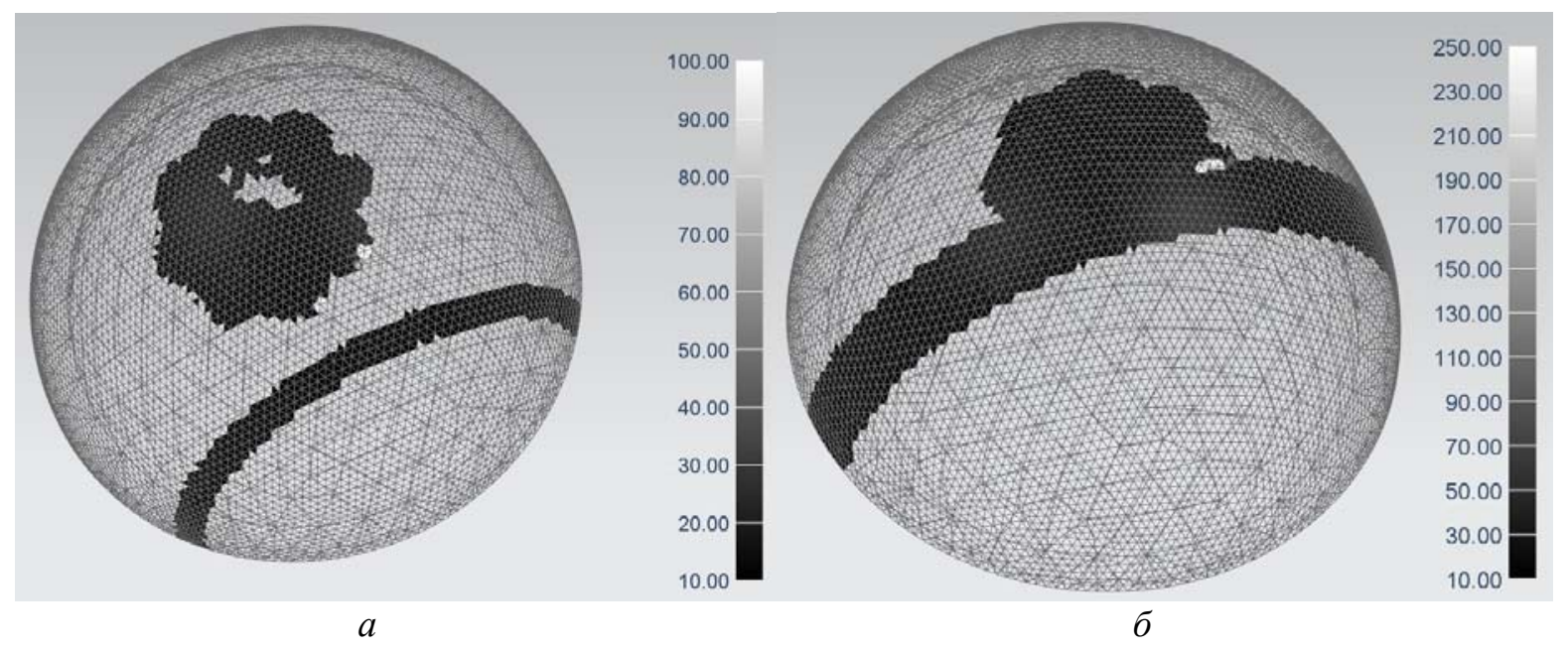

Рис. 6. Результаты расчетов критического усилия для чашки вертлужного компонента (распределение контактного давления (МПа) при различной степени недопокрытия): $a-23 \%$; $\sigma-33 \%$

компонента, при увеличении процента недопокрытия эти области (пятно в центре и полоса) приближаются друг к другу вплоть до полного объединения (см. рис. 6, б). Таким образом, чашка при недопокрытии до $30 \%$ имеет две области опоры в вертлужной впадине, из-за чего необходимая сила для поворота чашки выше, чем когда область опирания чашки одна. На основании этих рассуждений можно предположить, что чашка будет проворачиваться в вертлужной впадине, что согласуется с экспериментальными данными $[13,17,22,24,25]$.

Проводились расчеты для чашек различного диаметра (52, 54, 56 мм), результаты расчетов критической величины силы приведены на графике (см. рис. 7). При недопокрытии до $18 \%$ под действием силы до $4000 \mathrm{H}$ потери несущей способности вертлужного компонента не наблюдалось, в диапазоне недопокрытия от 18 до 30 \% величина критической силы превышала 2000 Н, в диапазоне от 30 до 40 \% критическая сила превышала $1000 \mathrm{H}$, с дальнейшим увеличением процента недопокрытия величина критической силы падала, вплоть до $500 \mathrm{H}$.

Совместно с оценкой перемещений чашки относительно вертлужной впадины производилась оценка напряженно-деформированного состояния костной ткани в области эндопротезирования, оценивались максимальные касательные напряжения, проверялся критерий разрушения костной ткани $[1,2]$. Результаты этой оценки показали, что коэффициент запаса по максимальным касательным напряжениям составил порядка 2-3 для высокого процента недопокрытия (свыше 20\%), в этом случае несущая способность терялась ввиду перемещения чашки; при низком проценте недопокрытия (до 20 \%) максимальные касательные напряжения превышали допустимые (при нагрузках свыше 4 кН).

Для оценки адекватности полученных результатов в расчетах было проведено сравнение с экспериментальными исследованиями [13, 17] (см. рис. 7), разница в расчетных величинах критической силы составила порядка $1000 \mathrm{H}$, при этом расчетные величины превышали данные из эксперимента. Процент отклонения при этом был более $35 \%$. Указанное отличие можно объяснить применяемым критерием потери несущей способности, в экспериментах было отмечено, что после первой (локальной) потери несущей способности чашка под действием внешних сил занимает новое положение и окончательная потеря несущей способности происходит уже для этого положения $[13,17]$. Отдельно стоит отметить произвольность выбора величины коэффициента трения, что также вносит большой вклад в определяемые величины критических сил. 
На основании известных диаграмм относительной (к весу тела) реакции в тазобедренном суставе $[2,10,14]$, возникающих при ходьбе, авторами был введен коэффициент динамичности, он принимался равным обратной величине максимального значения реакции (отнесенной к весу тела), возникающей в тазобедренном суставе при движении. Для пересчета найденной критической силы в допустимую массу $m$ пациента использовалась формула

$$
m=\frac{k_{D}}{g} P,
$$

где $k_{D}$ - коэффициент динамичности, принимался равным 0,$377 ; g$ - ускорение свободного падения, принималось равным 9,8 м/с ${ }^{2} ; P$ - величина критической силы.

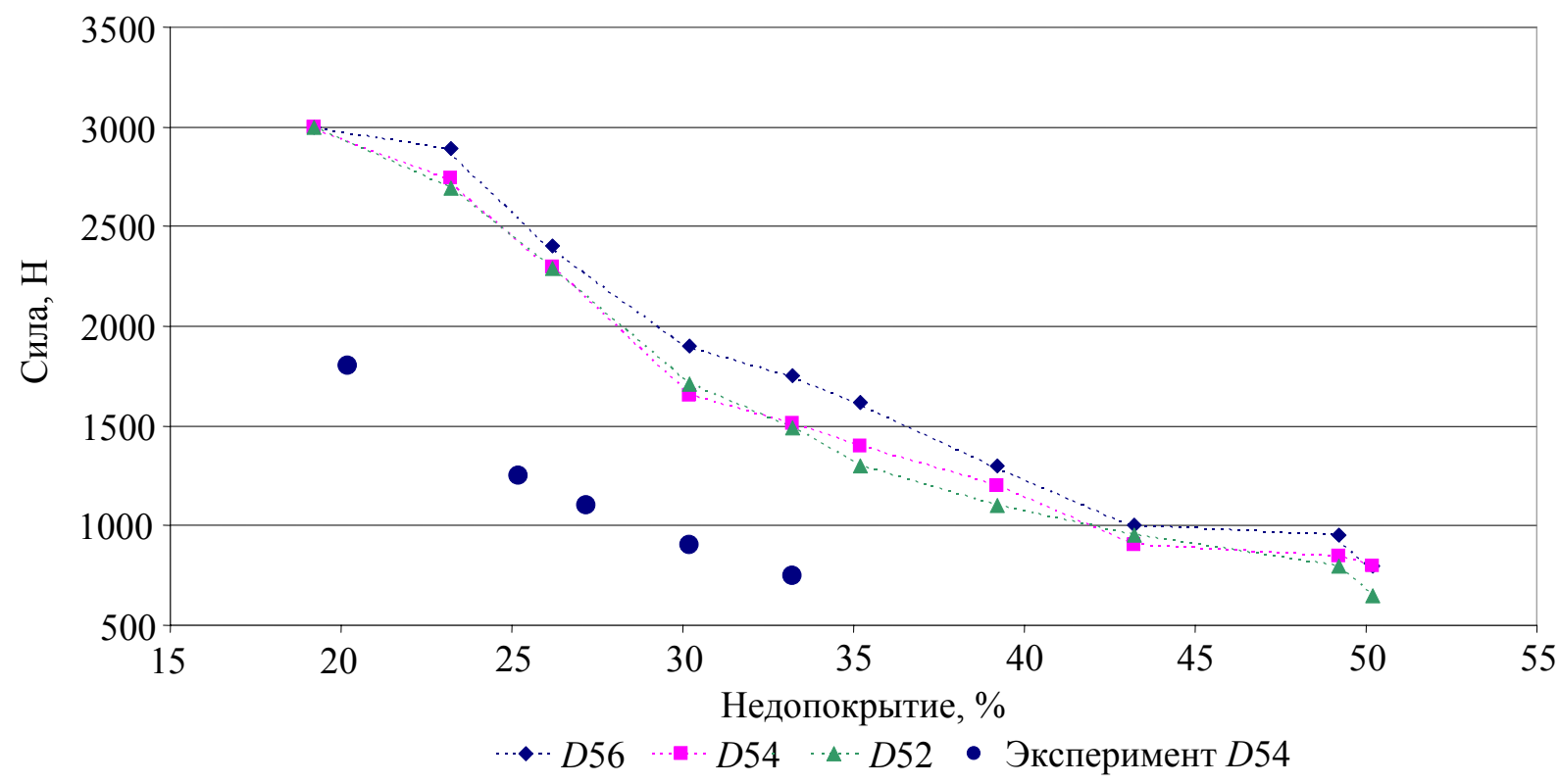

Рис. 7. Результаты расчетов критического усилия для чашки вертлужного компонента разного диаметра (ромб - 56 мм, квадрат - 54 мм, треугольник 52 мм) и результаты эксперимента для чашки диаметром 54 мм

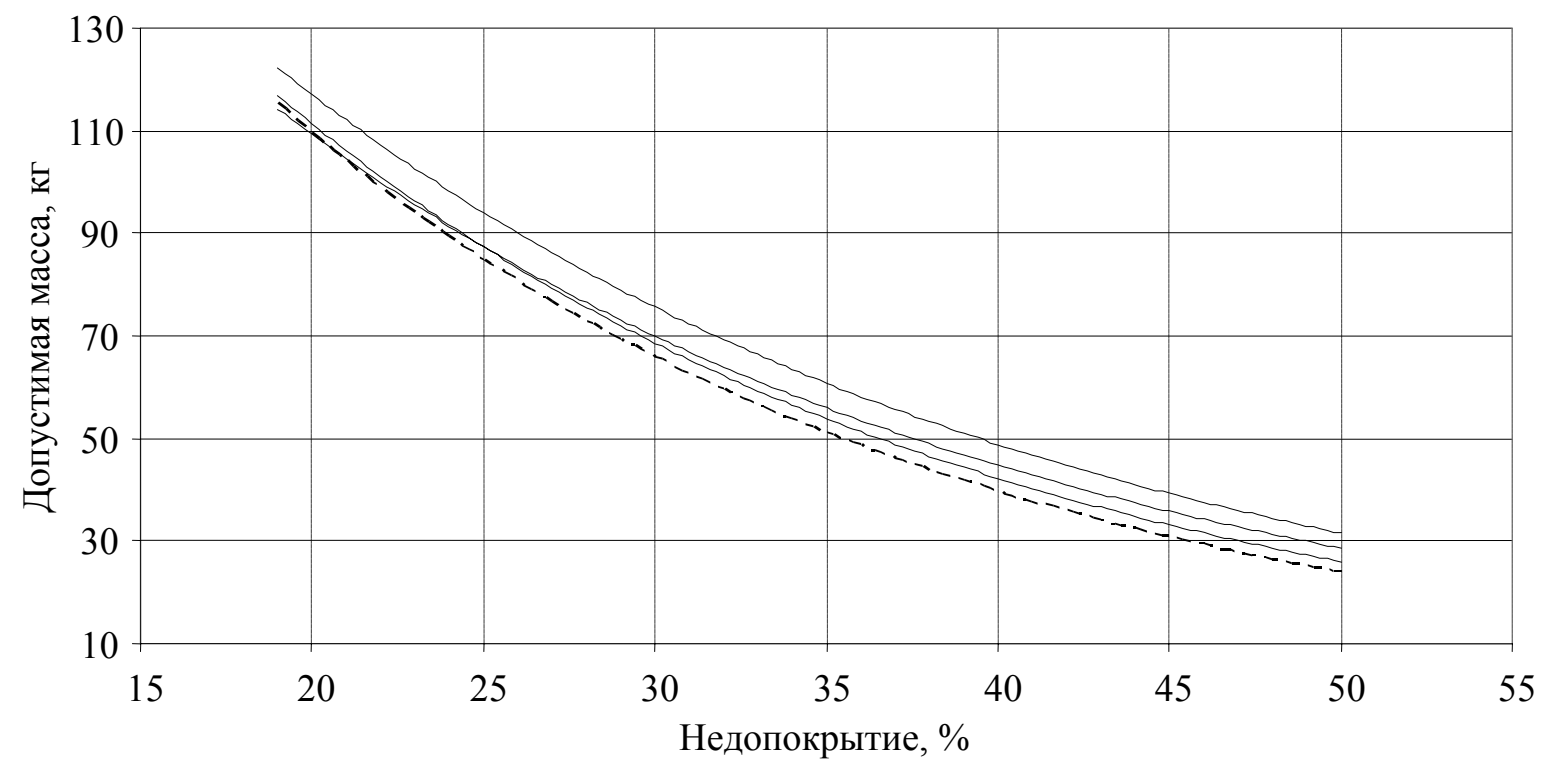

Рис. 8. Экспоненциальная зависимость допустимой массы от процента недопокрытия 
Полученные зависимости хорошо описываются уравнениями экспоненциальной регрессии, для получения удобной с точки зрения практики диаграммы были получены функции регрессий для расчетных данных для различных диаметров чашки:

$$
\begin{gathered}
y_{D 56}=280,39 e^{-0,0437 x}, \text { достоверность аппроксимации составила } R_{D 56}^{2}=0,9819, \\
y_{D 54}=266,9 e^{-0,0446 x}, \text { достоверность аппроксимации составила } R_{D 54}^{2}=0,9735, \\
y_{D 52}=293,74 e^{-0,0485 x}, \text { достоверность аппроксимации составила } R_{D 52}^{2}=0,9854 .
\end{gathered}
$$

На рис. 8 показаны полученные функции. Была определена нижняя граница для критических величин массы тела в зависимости от процента недопокрытия (пунктирная кривая на рис. 8), описывающее ее уравнение приведено ниже:

$y_{\mathrm{inf}}=302,09 e^{-0,0508 x}$, достоверность аппроксимации составила $R_{\mathrm{inf}}^{2}=0,9839$.

Допустимые соотношения веса пациента и степени дисплазии для операции эндопротезирования должны лежать ниже нижней границы экспоненциальной кривой (штрихованная линия на рис. 8).

\section{Выводы}

1. В работе приведены результаты численных исследований трехмерной модели таза с установленным ацетабулярным компонентом и моделируемой дисплазией. Моделировалось контактное взаимодействие имплантата и костной ткани. Полученные исследования позволили оценить зависимость между критической величиной силы и процентом недопокрытия ацетабулярного компонента эндопротеза, при которой возможна его имплантация без использования дополнительной опоры. Для использования полученных результатов в хирургической практике были построены экспоненциальные зависимости в осях «вес-величина недопокрытия в процентах». Полученная зависимость величины допустимого недопокрытия от массы пациента позволяет хирургу индивидуально и объективно оценить возможность установки ацетабулярного компонента эндопротеза.

2. При моделировании принимались следующие допущения: контактное взаимодействие считалось линейным; силы, действующие на тазовый компонент, считались постоянными по направлению; дисплазия вертлужной впадины моделировалась сферическим вырезом; указанные допущения продиктованы необходимостью упрощения математической модели с сохранением ее адекватности.

3. В целом полученные авторами результаты согласуются с данными литературы о том, что критическая величина недопокрытия составляет около $30 \%$ площади поверхности [17-26, 28] и что минимально необходимое покрытие чашки должно составлять не менее $70 \%$ площади ее поверхности [15]. Количественное сравнение с полученными результатами в рамках экспериментальных и клинических исследований показало, что разница в определяемых величинах критических сил составляет порядка $1000 \mathrm{H}$ [13, 15, 17], относительная величина составила $35 \%$. Указанное отличие можно объяснить применяемым критерием потери несущей способности, в экспериментах было отмечено, что после первой (локальной) потери несущей способности чашка под действием внешних сил занимает новое положение, и окончательная потеря несущей способности происходит уже для этого положения и зависит от произвольного выбора величины коэффициента трения. Поэтому необходимо определить величину коэффициента трения в специальном эксперименте. Остается открытым вопрос учета в математической модели особенностей геометрии 
чашки тазового компонента и механизма передачи усилий с бедренного компонента на тазовый через контактную пару эндопротеза. Для этого необходимы дополнительные экспериментальные исследования.

4. Полученные результаты не являются абсолютно бесспорными, поскольку реальные условия во время хирургического вмешательства могут отличаться от заложенных в математическую модель, но проведенные исследования позволяют сформировать представление о влиянии величины дефицита покрытия и помогают хирургу ориентироваться в вопросах выбора дополнительных методов стабилизации. Полученные зависимости критического веса и величины недопокрытия в процентах позволяют быть более свободным в выборе хирургической тактики при имплантации вертлужного компонента и более точно судить о степени недопокрытия вертлужного компонента как об одном из факторов, влияющих на механическую стабильность имплантата.

\section{СПИСОК ЛИТЕРАТУРЫ}

1. Акулич А.Ю., Акулич Ю.В., Денисов А.С. Экспериментальное определение разрушающих касательных напряжений трабекулярной костной ткани головки бедра человека // Российский журнал биомеханики. - 2010. - Т. 14, № 4 (50). - С. 7-16.

2. Акулич Ю.В., Подгаец Р.М., Скрябин В.Л., Сотин А.В. Исследование напряженнодеформированного состояния эндопротезированного тазобедренного сустава // Российский журнал биомеханики. - 2007. - Т. 11, №4. - С. 9-35.

3. Балафендиева И.С., Бережной Д.В., Секаева Л.Р. Исследование деформирования элементов транспортных сооружений, взаимодействующих с грунтом сложной физической природы // Морские интеллектуальные технологии. - 2011. - № 3. - С.81-84.

4. Голованов А.И., Коноплев Ю.Г., Султанов Л.У. Численное исследование конечных деформаций гиперупругих тел. IV. Конечноэлементная реализация. Примеры решения задач // Ученые записки Казанского университета. Серия: Физико-математические науки. - 2010. - Т. 152, № $4 .-$ C. $115-126$.

5. Голованов А.И., Султанов Л.У. Большие вязкоупругопластические деформации трехмерных тел // Ученые записки Казанского университета. Серия: Физико-математические науки. - 2005. - Т. 147, № 3. - С. 75-89.

6. Егоров Д.Л., Кузнецов С.А. Исследование контактного взаимодействия круглых пластин со штампами на основе численно-аналитической методики // Ученые записки Казанского университета. Серия: Физико-математические науки. - 2010. - Т. 152, № 4. - С. 127-134.

7. Егоров Д.Л., Кузнецов С.А. Численно-аналитическое решение задачи контактного взаимодействия круглой пластины и штампов различных форм // Вестник Казанского технологического университета. - 2013. - Т. 16, № 11. - С. 36-39.

8. Зайцева Т.А., Коноплев Ю.Г., Митряйкин В.И., Саченков О.А. Математическое моделирование вывиха имплантата в тазобедренном суставе // Вестник КГТУ им. А.Н.Туполева. - 2013. - № 1. C. 99-104.

9. Зайцева Т.А., Коноплев Ю.Г., Митряйкин В.И., Саченков О.А. Математическое моделирование установки ацетабулярного компонента с недопокрытием // Научно-технический вестник Поволжья. - 2014. - № 4. - С. 22-25.

10. Закиров Р.Х., Коноплев Ю.Г., Митряйкин В.И., Саченков О.А. Математическое моделирование биомеханики сустава // Научно-технический вестник Поволжья. - 2012. - № 1. - С.31-37.

11. Закиров Р.Х., Зарипов Р.А., Коноплев Ю.Г., Митряйкин В.И., Саченков О.А. Диагностика аспетического некроза головки бедренной кости с использованием спиральной компьютерной и магнитно-резонансной томографии и применение математического моделирования при планировании операции по эндопротезированию тазобедренного сустава // Практическая медицина. Актуальные проблемы медицины. - 2012. - Т. 2, № 8 (64). - С.63-68.

12. Каюмов Р.А., Шакирзянов Р.А., Шакирзянов Ф.Р., Богачев М.И. Адаптация методов строительной механики к моделированию поведения челюсти с имплантатом // Известия Казанского государственного архитектурно-строительного университета. - 2013. - № 2 (24). - С. 340-347.

13. Коноплев Ю.Г., Мазуренко А.В., Митряйкин В.И., Саченков О.А., Тихилов Р.М. Экспериментальное исследование влияния степени недопокрытия вертлужного компонента на несущую способность эндопротеза // Российский журнал биомеханики. - 2014. - Т. 18, № 3. C. 333-344. 
14. Коноплев Ю.Г., Митряйкин В.И., Саченков О.А. Применение математического моделирования при планировании операции по эндопротезированию тазобедренного сустава // Ученые записки Казанского университета. Серия: Физико-математические науки. - 2011. - С. 76-83.

15. Мазуренко А.В., Тихилов Р.М., Шубняков И.И., Плиев Д.Г., Денисов А.О. Безопасное эндопротезирование при диспластическом коксартрозе // Риски в современной травматологии и ортопедии: материалы межрегиональной научно-практической конференции с международным участием, посвященной памяти профессора А.Н. Горячева, 26-27 апреля 2013. - Омск, 2013. C. 38 .

16. Неверов В.А., Закари С.М. Ревизионное эндопротезирование тазобедренного сустава. - СПб.: Образование, 1997. - 112 с.

17. Тихилов Р.М., Шубняков И.И., Мазуренко А.В., Митряйкин В.И., Саченков О.А., Кузин А.К., Денисов А.О., Плиев Д.Г., Бояров А.А., Коваленко А.Н. Экспериментальное обоснование установки ацетабулярного компонента с недопокрытием при эндопротезировании пациентов с тяжелой степенью дисплазии // Травматология и ортопедия России. - 2013. - № 4 (70). - С. $42-51$.

18. Atilla B. Position of the acetabular component determines the fate of femoral head autografts in total hip replacement for acetabular dysplasia // J. Bone Joint Surg. - 2007. - Vol. 89, № 7. - P. 874-878.

19. Bicanic G., Delimar D., Delimar M., Pecina M. Influence of the acetabular cup position on hip load during arthroplasty in hip dysplasia // Int. Orthop. - 2009. - Vol. 33, № 2. - P. 397-402. DOI: 10.1007/s00264008-0683-z.

20. Delbarre J.C. Total hip arthroplasty after proximal femoral osteotomy: 75 cases with 9-year follow-up // Rev. Chir. Orthop. - 2002. - Vol. 88, № 3. - P. 245-256.

21. Engesaeter L.B., Furnes O., Havelin L.I. Developmental dysplasia of the hip - good results of later total hip arthroplasty: 7135 primary total hip arthroplasties after developmental dysplasia of the hip compared with 59774 total hip arthroplasties in idiopathic coxarthrosis followed for 0 to 15 years in the Norwegian Arthroplasty Register // J. Arthroplasty. - 2008. -Vol. 23, № 2. - P. 235-240. DOI: 10.1016/j.arth. 2007.03.023.

22. Garvin K.L., Bowen M.K., Salvati E.A., Ranawat C.S. Long-term results of total hip arthroplasty in congenital dislocation and dysplasia of the hip: a follow-up note // J. Bone Joint Surg. Am. - 1991. Vol. 73. - P. 1348-1354.

23. Golovanov A.I., Sultanov L.U. Postbuckling elastoplastic state analysis of three-dimensional bodies taking into account finite strains // Russian Aeronautics. - 2008. - Vol. 51, № 4. - P. 362-368. DOI: 10.3103/S106879980804003X

24. Gross A.E., Allan D.G., Catre M., Garbuz D.S., Stockley I. Bone grafts in hip replacement surgery: the pelvic side // Orthop. Clin. North. America. - 1993. - Vol. 24. - P. 679-695.

25. Jasty M., Anderson M.J., Harris W.H. Total hip replacement for developmental dysplasia of the hip // Clin. Orthop. Relat. Res.- 1995. - Vol. 311. - P. 40-45.

26. Rogers B.A., Garbedian S., Kuchinad R.A. Total hip arthroplasty for adult hip dysplasia // J. Bone Joint Surg. Am. - 2012. - Vol. 94. -P. 1809-1821.

27. Sachenkov O.A., Mitryaikin V.I., Zaitseva T.A., Konoplev Yu.G. Implementation of contact interaction in a finite-element formulation // Applied Mathematical Sciences. - 2014. - Vol. 159, № 8. - P. 7889-7897. DOI: 10.12988/ams.2014.49769.

28. Thillemann T. Implant survival after primary total hip arthroplasty due to childhood hip disorders: results from the Danish Hip Arthroplasty Registry // Acta Orthop. - 2008. - Vol. 79, № 6. - P. 769-776. DOI: $10.1080 / 17453670810016830$.

\title{
NUMERICAL STUDY OF THE INFLUENCE OF THE DEGREE OF UNDERCOVERAGE OF THE ACETABULAR COMPONENT ON THE LOAD-BEARING CAPACITY OF HIP JOINT ENDOPROSTHESIS
}

\author{
Yu.G. Konoplev (Kazan, Russia), A.V. Mazurenko (Cheboksary, Russia), \\ O.A. Sachenkov (Kazan, Russia), R.M. Tikhilov (Saint-Peterburg, Russia)
}

The aim of the work is to estimate the critical value of undercoverage of the acetabular component, which allows its implantation without using additional support. The relevance of this study is determined by the clinical practice and the absence of clear recommendations on the tactics of endoprosthesis replacement with an undercoverage of the 
acetabular component. Numerical study is carried out with the help of finite element method in Siemens NX. The degree of dysplasia is estimated on the basis of the original method of intraoperational measurement of undercoverage of the acetabular component. The calculations for the cups of 52,54, and $56 \mathrm{~mm}$ in diameter are made and take into consideration the influence of contact interaction. Finite element method has been used to solve the problem of stress-strain state of the body. Numerical algorithm of implementation of contact interaction is based upon an iteration process of determining the contact zone. Based on the results of the calculations, the mechanism of loss of the load-bearing capacity of the acetabular component is studied and the dependence of the values of critical reaction in a joint on the values of undercoverage are determined. The critical values of joint reaction stand for the minimum force at which the loss of load-bearing capacity takes place and is identified with the appearance of relative sliding in the contact area. The authors determine stability of implants at the extent of up to $20 \%$ of undercoverage, the value of critical force exceeds $2 \mathrm{kN}$ in the range between 20 and $30 \%$ and decreases with the increasing percentage of undercoverage. The obtained data is compared with clinical and experimental data; the difference in the determined values constituted around $1 \mathrm{kN}$ (around $35 \%$ in relative values). Based on the obtained results, the exponential approximation of the relationship between the value of critical reaction in the joint and various degrees of undercoverage is calculated. The calculated dependence of the admissible undercoverage on the body weight allows a surgeon to assess the possibility of insertion of the acetabular component of endoprosthesis individually and objectively.

Key words: hip replacement, dysplasia, contact interaction, mathematical simulation. 